Eur. J. Psychiat. Vol. 28, N. ${ }^{\circ}$ 1, (50-65)

2014

Keywords: Happiness; Quality of Life; Wellbeing;

Functioning; Health terminology; Nomenclature.

\title{
Use of the terms "Wellbeing" and "Quality of Life" in health sciences: A conceptual framework
}

\author{
Luis Salvador-Carulla*,** \\ Ramona Lucas ${ }^{\star * *, * \star * *}$ \\ José Luis Ayuso-Mateos ${ }^{\star \star \star \star \star *, \star \star \star \star \star \star,, \star \star \star \star \star \star \star \star ~}$ \\ Marta Miret ${ }^{\star \star \star \star \star \star, \star \star \star \star \star \star \star *, \star \star \star \star \star \star \star *}$
}

* Centre for Disability Research and Policy.

Faculty of Health Sciences T315a

Cumberland Campus. The University

of Sydney, Lidcombe

** Section of Classification, Diagnostic

Assessment and Nomenclature. World

Psychiatric Association, Geneva

*** Department of Methodology and

Behavioural Sciences, University of

Barcelona. SGR 822 Generalitat de

Catalunya, Barcelona

**** Cambridgeshire and Peterborough NHS

Foundation Trust, Cambridge

***** Instituto de Salud Carlos III, Centro de Investigación Biomédica en Red de Salud Mental, CIBERSAM, Madrid

****** Department of Psychiatry,

Universidad Autónoma de Madrid, Madrid

****** Hospital Universitario de La

Princesa, Instituto de Investigación

Sanitaria Princesa (IP), Madrid

AUSTRALIA

SPAIN

SWITZERLAND

UNITED KINGDOM

\footnotetext{
ABSTRACT - Background and Objectives: The assessment of wellbeing is a top priority in health sciences. The aim of this paper is to review the history of the concept of wellbeing and "Quality of Life" (QoL), and to understand the theories and assumptions that guided this field in order to provide a conceptual framework that may eventually facilitate the development of a formal synset (grouping of synonyms and semantically similar terms) of health-related wellbeing.
} 
Methods: The history of the concept of wellbeing and QoL was reviewed in order to provide a conceptual framework.

Results: Huge differences exist on the definition of "Wellbeing" and its relationship with QoL, "Happiness" and "Functioning" in the health context. From a dimensional perspective, health related wellbeing could be regarded as an overarching construct characterised by asymmetrical polarity, where "wellbeing" embeds the concept of "ill-being" as "health" incorporates de concept of "disease".

Conclusions: A common conceptual framework of these terms may eventually facilitate the development of a formal synset of health-related wellbeing. This terminological clarification should be part of a new taxonomy of health-related wellbeing based on the International Classification of Functioning, Disability and Health (ICF) framework that may facilitate knowledge transfer across different sectors and semantic interoperability for care management and planning.

Received: 25 November 2013

Revised: 2 January 2014

Accepted: 3 January 2014

\section{Introduction}

The assessment of "Wellbeing" is a top priority for governments, international organisations, health companies and research institutions alike. There is an increasing interest and hard competition to produce wellbeing indexes aimed at replacing current Gross Domestic Product (GDP) and burden measures, to measure wellbeing in population surveys and new models of care, and to design wellbeing policies and related health care interventions ${ }^{1}$. Wellbeing has also turned into an indispensable concept in epistemology to appropriately frame the complex models of disease, healthcare and human function. Regardless of its current success, huge differences persist on the definition of wellbeing and its relationship with happiness, satisfaction, mental health, Quality of Life (QoL), social capital, mental capital and human functioning. To a large extent these differences relate to the diverse backgrounds where this concept developed during the last 100 years. Although the link between wellbeing and health is in- escapable, two major areas of development could be traced outside the health sector: one is related to wellbeing and happiness assessment in demographics, psychology and education and another is related to the measurement of wellbeing in economics.

The aim of this paper is to review the history of the concept of wellbeing and QoL, and to understand the theories and assumptions that guided this field in order to provide a conceptual framework that may eventually facilitate the development of a formal synset (grouping of synonyms and semantically similar terms) of health-related wellbeing.

\section{The origins of Wellbeing in social sciences}

The evolution of the non-health related concept of wellbeing throughout the last century has been described by Erik Agner ${ }^{2}$. He traces it back its origin to the development of "eupathics" in the first quarter of the $20^{\text {th }}$ Century. This discipline was concerned with 
the study of the "well being of the normal", and it evolved into the social studies of happiness and marital success, apart from other areas of education, psychology, and personality studies in the 1920s and 1930s. A decade later Hornell $\mathrm{Hart}^{3}$ produced the "Chart for happiness" and a thermometric scale called "Euphorimeter". He also coined two complementary definitions of happiness, one related to subjective status and satisfaction (people are happy insofar as they are prone to saying sincerely that they are happy); and other one related to personal choice (people are happy insofar as they are in whatever state of consciousness that they seek to attain or maintain). Later on, Diener et $a l .{ }^{4}$ defined subjective wellbeing as "a broad category of phenomena that includes people's emotional responses, domain satisfactions, and global judgments of life satisfaction".

The economic approach to wellbeing dates back to the 1930's when Simon Kuznets ${ }^{5}$ argued that a nation's welfare can scarcely be inferred just from its national income an advocated for an assessment of wellbeing. In the 1970's economists constructed metrics to measure societal wellbeing in terms of utility as expressed in "revealed preferences" that were later used in the assessment of QoL in health economics ${ }^{6}$. Tversky and Kahne$\operatorname{man}^{7}$ contributed to the understanding of irrational choice, and later on to its relation to happiness and wellbeing ${ }^{8}$. The work of Daniel Kahneman separated wellbeing into two complementary conceptualisations: "experienced wellbeing", which is typically captured by measures of positive affect, pleasure, and happiness, and their opposites negative affect, misery, or distress; and "evaluative wellbeing", which captures judgments of overall life satisfaction or fulfilment in selected domains such as autonomy, personal growth, and purpose in life.

\section{Beyond Gross Domestic Product (GDP)}

In 1995 Cliff Cobb developed the Genuine Progress Indicator (GPI), a measure of sustainable economic wellbeing designed to indicate progress in people's QoL and economic, social, and environmental wellbeing. The GPI attempted to address the limitations of GDP by measuring the social and environmental costs and benefits that the GDP ignores or counts as progress ${ }^{9}$. Anielski ${ }^{9}$ defined Genuine Wealth as: "the conditions of wellbeing that are true to our core values of life".

The European Commission ${ }^{10}$ published a communication which acknowledged the limitations of GDP and proposed to complement GDP with environmental and social indicators such as a comprehensive environmental index and measures of QoL and wellbeing. In the same line, the Commission on the Measurement of Economic Performance and Social Progress proposed several recommendations on how to better measure economic performance, societal wellbeing and sustainability, and recommended that the measurement system should shift emphasis from measuring economic production to measuring people's wellbeing ${ }^{11}$. A few months later, the British government asked the Office of National Statistics to devise a new way of measuring wellbeing in Britain in order to start measuring the progress as a country, not just by how the economy is growing, but by how the people's lives are improving ${ }^{12}$.

These initiatives are not limited to Europe. In Bhutan, the happiness of the population is considered as a public good, and it is the responsibility of the government to create an enabling environment for the pursuit of happiness, as reflected in the constitution ${ }^{13}$. The Centre for Bhutan Studies has developed a Gross National Happiness (GNH) index under nine domains: psychological wellbeing, 
health, education, time use, culture, governance, community vitality, environmental diversity, and living standards ${ }^{14}$.

In contrast to western societies, where happiness is conceptualised as a transitory mood state $^{15}$ or as the "predominance of the frequency of occurrence of positive emotional experiences over negative ones"16, within the GNH model, the concept of happiness has a broader meaning that refers to a state of being rather than an emotion or feeling. In western applications, the term "wellbeing" is more reflective of "happiness" within GNH. In this model, health is only a determinant of happiness ${ }^{15}$.

In 2011 the OECD Better Life Initiative launched an interactive index called Your Better Life Index [oecdbetterlifeindex.org] to measure wellbeing and progress. The index allows citizens to measure their lives in a way that goes beyond traditional GDP numbers and to compare lives across 34 countries based on 11 dimensions, giving their own weight to each of the dimensions.

The Gallup World Poll has been collecting information about wellbeing since 2006. The poll covers at least 130 countries in any given year. In each country a representative sample of the adult population is evaluated. In addition, in 2008 Gallup started a daily survey in the United States. The Gallup-Healthways Well-Being Index provides real-time measurement of health and wellbeing by interviewing at least 500 U.S. adults every day ${ }^{17}$.

\section{The origins of Wellbeing and QoL in health sciences}

The history of the terms Wellbeing and QoL in health sciences follows an intricate path that is not easy to disentangle. In the 1930s several voices started to recognise that health was beyond the absence of illness:
"Health must come first: the mere state of not being ill must be recognised as an unacceptable substitute, too often tolerated or even regarded as normal. We must, moreover, face the fact that while immense study has been lavished on disease no one has intensively studied and analysed health, and our ignorance of the subject is now so deep that we can hardly claim scientifically to know what health is" "18. And it was also at that time that the term "Happiness" was first used in the health sector in the UK" ${ }^{19}$. The term "Wellbeing" was incorporated to the definition of health at the WHO constitution following a proposal by Andrija Stampar, one of the pioneers of public health. It said health was "a state of complete physical, mental and social well-being and not merely the absence of disease or infirmity"20. This pivotal change over the pre-war conceptualisation of health and disease promoted the incorporation of "wellbeing" to the medical terminology, although it took other three decades before it was officially adopted at the Alma Alta declaration of 1978. Pubmed records the first appearance of this concept in a French paper on mental health published in $1955^{21}$.

This was followed by the incorporation of the "positive aspects of health" to public health surveys such as the US National Health Surveys since $1957^{22}$, and the addition of brief subjective measures of "positive" and "negative" wellbeing at the Bradburn's ${ }^{23}$ scale a decade later. During the following thirty years, "Wellbeing" was a concept closely intertwined to QoL in Medicine. Thus, QoL was defined as the subjective aspects of wellbeing, while wellbeing was assessed as a generic component of QoL. Surprisingly enough, efforts to reach an international consensus on the definition of wellbeing and its taxonomy have been minimal in comparison with QoL.

Prutkein and Feinstein ${ }^{24}$ have revised the origins of the concept of QoL and its evolution 
in Medicine until 1986. Although the first mention to QoL is identified in the field of Nephrology in the late 1960's, the standard assessment of QoL was preceded by the evaluation of daily living in time diaries since the 1890's later incorporated to the social sciences study of human behaviour ${ }^{25}$. Right after World War II, the Karnofsky index was introduced in healthcare to evaluate the performance status of cancer patients and persons with disabilities ${ }^{26}$, and this was followed by series of subsequent clinical indexes of "Activities of Daily Living"27.

Self-reported ratings were incorporated to the assessment of QOL in national surveys during 1970s. Four main domains or indexes were included: Overall Scale of Life Satisfaction, Index of Well-being, Index of General Affect, and Index of Perceived Stress ${ }^{28}$. Later on, global questions such as "How do you feel about your life as a whole?" were added to the questionnaires, and the basic QoL domains were also expanded ${ }^{29}$.

The "Vitagram" may be regarded as the first QoL scale ${ }^{30}$. It was developed in Sweden to assess the extent to which lung cancer patients enjoyed a meaningful life after treatment and it was inspired in the call made by Feinstein et al..$^{31}$ to develop QoL measures for cancer patients. Full QoL scales were developed during the late $1970 \mathrm{~s}^{32}$, and the topic boomed after the second half of the 1980s leading to the development of a whole array of generic and disease-specific scales, as well as specialised journals, international networks and scientific societies in this field ${ }^{33}$.

The QoL construct was adopted by WHO which in 1991 started a project to develop an instrument (the World Health Organization Quality of Life-WHOQOL-) for measuring QoL internationally. QoL was defined as "a person's perception of his/her position in life within the context of the culture and value systems in which he/she lives and in relation to his/her goals, expectations, standards, and concerns $^{34}$. The definition highlighted the view that QoL referred to a subjective evaluation, including both positive and negative dimensions, and which was embedded in a cultural, social, and environmental context ${ }^{35-37}$. The first WHOQOL field trial form (WHOQOL-100) contained 100 questions covering six domains and 24 facets $^{38,39}$. Domains represent a high level of organisation and facets are an intermediate level of organisation between domains and items. Each facet is represented by a set of four specific items. Next, the short version WHOQOL-BREF was developed for use in situations where time is limited and where respondent burden must be minimized ${ }^{40}$.

During the 2000's the WHOQOL Group developed several supplementary modules to be used in conjunction either with the WHOQOL-100 or WHOQOL-BREF: a module on Spirituality, Religiousness and Personal Beliefs $^{40}$, the WHOQOL-OLD module for older adults ${ }^{41}$, and the WHOQOL disability module (WHOQOL-DIS) for persons with physical and intellectual disabilities ${ }^{42}$.

\section{Non-canonical models of the construct QoL/Wellbeing}

To avoid including non-medical components in the QoL construct, some researchers decided to focus on what was called Health related Quality of Life (HRQoL): medical factors, expressed as a combination of functional status and symptoms related to specific diseases $^{24}$. As the interest on HRQoL and patient reported outcomes continues to grow and new domains of the human experience are incorporated into an increasingly complex QoL construct; the very same concept of HRQoL is being contested. 
While QoL was confirmed as a leading field in Medicine, key experts in the development of the HRQoL suggested that the evaluation should also include other non selfreported ratings such as physician-generated assessments of patients' performance status and ratings made by family members of the psychological and social health status of patients unwilling or unable to provide such information ${ }^{43}$. This statement challenged the very same concept of QoL adopted by the WHO. There was also an increasing awareness of the conceptual problems derived from the absence of theoretical models to guide the development of QoL measures ${ }^{43}$.

Prutkein and Feinstein ${ }^{24}$ attributed these problems and imperfections in QoL indexes to "an origin in two different streams of thought, neither of which has led to a fully satisfactory approach" as they were not originally designed for appraising the particular personal distinctions of the way people feel about their own QoL. They identified a medical (clinical) stream based on single measures of health status (not a suitable way to denote a patient's belief about QoL); and a psychosocial stream (actually public health) based on multi-item instruments (not suitable to allow patients to express changes in status). They conclude: "Since quality of life is determined uniquely by each patient, and reflects a personal reaction rather than an objective "status", a possible solution to the problems is to return to an old clinical approach, which directly asks patients to indicate what they feel".

On the other hand, the incorporation of non self-reported information to the QoL construct and the inclusion of domains of life related to contextual wellbeing in the WHOQOL system, indicates that the boundaries between QoL and Wellbeing are increasingly blurred, and that an idly defined QoL/Wellbeing construct is used both in clinical practice and in public health assessment.
The underlying conceptual problems of QoL/Wellbeing measurement are actually far more complex, as other separate sources and models can be identified when the QoL/Wellbeing construct is considered. Apart from the clinical and public health perspectives previously mentioned, it is necessary to take into account the economical, socio-cultural, psychobiological, psychological and functional approaches to QoL/Wellbeing.

\section{The health economics model of QoL}

Health economists' perspective on QoL differs significantly from that developed in the medical sector ${ }^{6,44}$. While in Medicine HRQoL is a status function, in health economics it is a utility function and it is related to personal choice. In the first case, a subjective rating scale provides information about the patient's present status in one or in several dimensions related to the QoL construct; while in health economics, the assessment is based on the individual's preference between two health states.

This is related to the "expected utility theory", formalised by von Neumann and Morgenstern in the late 1940s, where every result of a choice between two alternatives (preference) produces a certain degree of wellbeing or utility. Subjective utility (the satisfaction derived from the alternative chosen), differs from the objective expected monetary gains on several points, such as risk assumption, choice certainty, degree of probability, or previous experience and cognition. For example, choices concerning gains are associated with risk aversion, while those concerning losses are associated with risk assumption, even when the expected monetary changes are equal for both choices ${ }^{44}$.

Weinstein and Stason ${ }^{45}$ developed the costutility analysis and a related index called the 
QALY (Quality Adjusted Life Years) to assess the net effectiveness of an alternative in question (e.g. a new drug) expressed by the tradeoffs between additional survival and QoL. QALYs can be calculated by different procedures and there are further questions regarding whether it is feasible to estimate monetary values of a $\mathrm{QALY}^{46}$. An unscalable consequence has been that the same name (HRQoL) and the same rating scales (e.g. SF-36, EQ5D) have been used for completely different purposes by clinicians (measure of health status) and health economists (measure of health preference and related QALYs) ${ }^{6}$.

\section{The Psychosocial - cultural models of QoL and Wellbeing}

The trend to broaden the QoL construct and to increase its complexity could be seen in other areas of psychology and social sciences where an emphasis was given to the positive aspects of this construct. In contrast with the health sector, the assessment of wellbeing and QoL in social sciences followed formal models of wellbeing to design related 'Wellbeing' or 'QoL' rating scales. The best known approach to the psychology of wellbeing is the positive psychology movement ${ }^{47}$; however other psychosocial and cultural models developed a decade earlier deserve attention.

Carol Ryff ${ }^{48}$ published the Scales of "Psychological Well-being" (PWB). PWB is grounded in the theoretical framework of the personal self and identity previously developed in social psychology ${ }^{48,49}$. It defines "psychological wellbeing" as a micro-level construct that conveys information about how individuals evaluate themselves and the quality of their lives. This theory-driven, multidimensional formulation of psychological wellbeing goes beyond the absence-of-illness criteria and "derives from life-span de- velopmental theories which formulate the unfolding tasks and challenges of human growth; clinical accounts of what it means to be self-actualized, mature, fully functioning, or individuated; and formulations of positive criteria of mental health" ${ }^{50}$. This positive assessment of Wellbeing applied initially in the field of ageing has been broadly tested in relation to several biomarkers of mortality ${ }^{50}$ and allostatic load. The six personal factors can be divided in two main domains: hedonic (related to feeling of pleasure) and eudemonic psychological wellbeing. Eudemonic wellbeing evolves from pursuing contexts and relationships that fulfil intrinsic human needs while continually extending the self, resulting in personal growth ${ }^{51}$. This and other models of happiness and wellbeing have driven to the new field of positive psychology ${ }^{52}$. This psycho-social model of wellbeing has been expanded to incorporate a biological component to explain the link between these facets and longevity (stimulation of enrichment of dendritic networks in the hippocampus and spine retraction in the basolateral amygdala; and the possibility that the release of intracerebral oxytocin is a mediating factor between persistently supportive social environments and reduced disease in later life, as exemplified by low levels of allostatic load $)^{53}$.

Robert Schalock and colleagues ${ }^{54}$ developed a "Quality of Life Index" for intellectual disabilities within the context of the positive approach to this entity. The American Association of Intellectual and Developmental Disabilities (AAIDD), former American Association of Mental Retardation (AAMR) formalised the AAMR/AAIDD multidimensional model of human functioning. Shalock's underlying social concept of QoL was completely different from HRQoL. It defined QoL as "a multidimensional construct that has both subjective and objective components and is influenced by personal and environmental factors" 55 . Shalock's scale was further deve- 
loped and progressively became a comprehensive multidimensional model of "QoL" for persons with disabilities.

The Multicultural Quality of Life Index (MQLI) is an example of the assessment of QoL from a transcultural perspective. This culturally sensitive rating scale incorporates basic domains from WHODAS-brief and support (social-emotional and community and services support). To a limited extent the cultural dialogical approach ${ }^{56}$ has incorporated the experience of feelings to the self-reported QoL/Wellbeing. Examples are feelings/experiences of "unsureness" 57 , "feeling understood/being listened" 58 , and suffering ${ }^{59}$.

\section{The psychobiological models of QoL/Wellbeing}

Jeffrey Gray ${ }^{60}$ developed a theory of anxiety based on a biological model of personality related to the introversion-extroversion domain and its relation to the activation and inhibition systems of the brain. This and other related models provided ground for a more refined biological theory of personality that included four basic temperamental traits, three character domains of self-concept as well as a related assessment instrument, the Temperament and Character Inventory (TCI). The relation of these traits to self-awareness of the person as an autonomous individual, an integral part of humanity and of the universe as a whole, was formulated since the publication of this model ${ }^{61}$. Nevertheless, its formal relationship to wellbeing and negative/positive affect was established quite recently ${ }^{62,63}$. The links of this model with culture have also been established. While Self-directedness and Cooperativeness are positively associated with wellbeing regardless of culture, Self-transcendence increases positive affect but, based on culture, it can also increase negative affect ${ }^{64}$.
On the one hand, these findings on wellbeing based on bio-psychological personality traits (TCI) seem to converge with the approach to QoL based on individual status (WHOQOL-SRPB) ${ }^{40}$, as they identify highly complex personal domains (self-awareness, spirituality) as key components of the QoL/ Wellbeing construct. These personal facets seem to play a more relevant role than the traditional components of QoL in wellbeing. On the other there is a tendency to expand these models to incorporate aspects from other fields in order to develop integrative or holistic approaches which may incorporate biological, psychological, social, and cultural aspects as well as preferences, experiences and rights.

\section{Holistic/integral approaches to wellbeing}

Understanding the complexity of the QoL/Wellbeing construct leads to accepting the complexity of its evaluation, the need to decompose its facets, the little usability of the traditional QoL construct, and the need to reformulate the concept of Health related Wellbeing (HrWB) from an integrative perspective. To date, two approaches have attempted to provide a holistic approach to HrWB: the ICF model of health functioning and the person integrated diagnosis model of personcentered medicine ${ }^{65}$.

\section{The WHO approach to HrWB}

During the last two decades the WHO has set up a framework for the conceptualisation and analysis of HrWB and has clarified the existing relations between health and disease, provided a life-span approach and a positive health perspective ${ }^{66-70}$. These reports 
emphasise positive mental health and social capital as well as a cultural sensitive approach that takes into account the resources available across countries. In any case the main WHO document to frame Wellbeing and related concepts is the International Classification of Functioning, Disability and Health $(\mathrm{ICF})^{71}$, which can be regarded as a first attempt to develop a unified and standard language for health and related problems.

Since its release the ICF has progressively acquired a central role at the WHO Family of International Classifications, as the logical connexion between the ICD subgroup (International Classification of Diseases) and the classifications of contextual factors such as the International Classification of Health Interventions (ICHI) ${ }^{72}$ or health services at the System of Health Accounts ${ }^{73}$.

ICF is indeed a proto-taxonomy of the different components (determinants, factors and consequences) of health conditions ${ }^{74}$. A relevant unforeseen aspect of ICF is that it is both a classification of "Health-related Functioning" (HrF) and the conceptual framework of the WHO HrF construct and related entities such as Health related Wellbeing. These two roles of ICF do not always coincide; and a major problem was the lack of a formal ontology approach when it was developed. As a consequence, the classification is incomplete and not fully congruent ${ }^{75}$.

The on-going ICF review process has included ontology as an essential component incorporating a specific working group on the ontology of ICF at the Functioning and Disability Reference Group (FDRG) committee. There is also a link between ICF and the International Health Terminology Standards and Development Organisation (IHTSDO) and the related Systematized Nomenclature of Medicine-Clinical Terms (SNOMED CT) system. Among other ontology groups, the
OntoICF group developed the ontology of this system ${ }^{76}$ within the MURINET network (Multidisciplinary Research Network on Health and Disability in Europe).

Although we still lack enough information to build a formal ontology of ICF, it is possible to define HrWB and its related meta-concepts within the WHO framework. Figure 1 shows a preliminary conceptual map of Health related Wellbeing, Health related Conditions and Health-related Functioning and disability using different perspectives from WHO.

This schema indicates that concepts usually regarded as the two poles of a unidimensional bipolar construct (i.e. Health conditions and Disease, Health related Functioning and Disability) are in fact asymmetric. Functioning and Disability are two related domains of the construct "Health-related Functioning". In this construct there is a clear asymmetry between the poles, as positive functioning involves many more alternatives than negative functioning-disability. On the other hand, the analysis of the hierarchical structure and the conceptual relationship between the terms "functioning" and "disability" at the WHO family of classifications and related documents may indicate that "disability" is actually a subcategory of "functioning", as "disease" is a subcategory of "health condition" [Fig. 1]. The current definition of "functioning" at ICF should incorporate the wording "positive functioning" and "health-related" functioning as the global term, as HRQoL is regarded as a subcategory of wellbeing.

The broad "WHO-ICF" perspective on HrWB provided at Figure 1 has a significant implication also for HRQoL. The development of ICF and its conceptual approach has challenged to a certain extent the WHOQOL traditional construct. ICF was first considered a complementary to QoL as it contributed to the assessment of the objective aspects of 

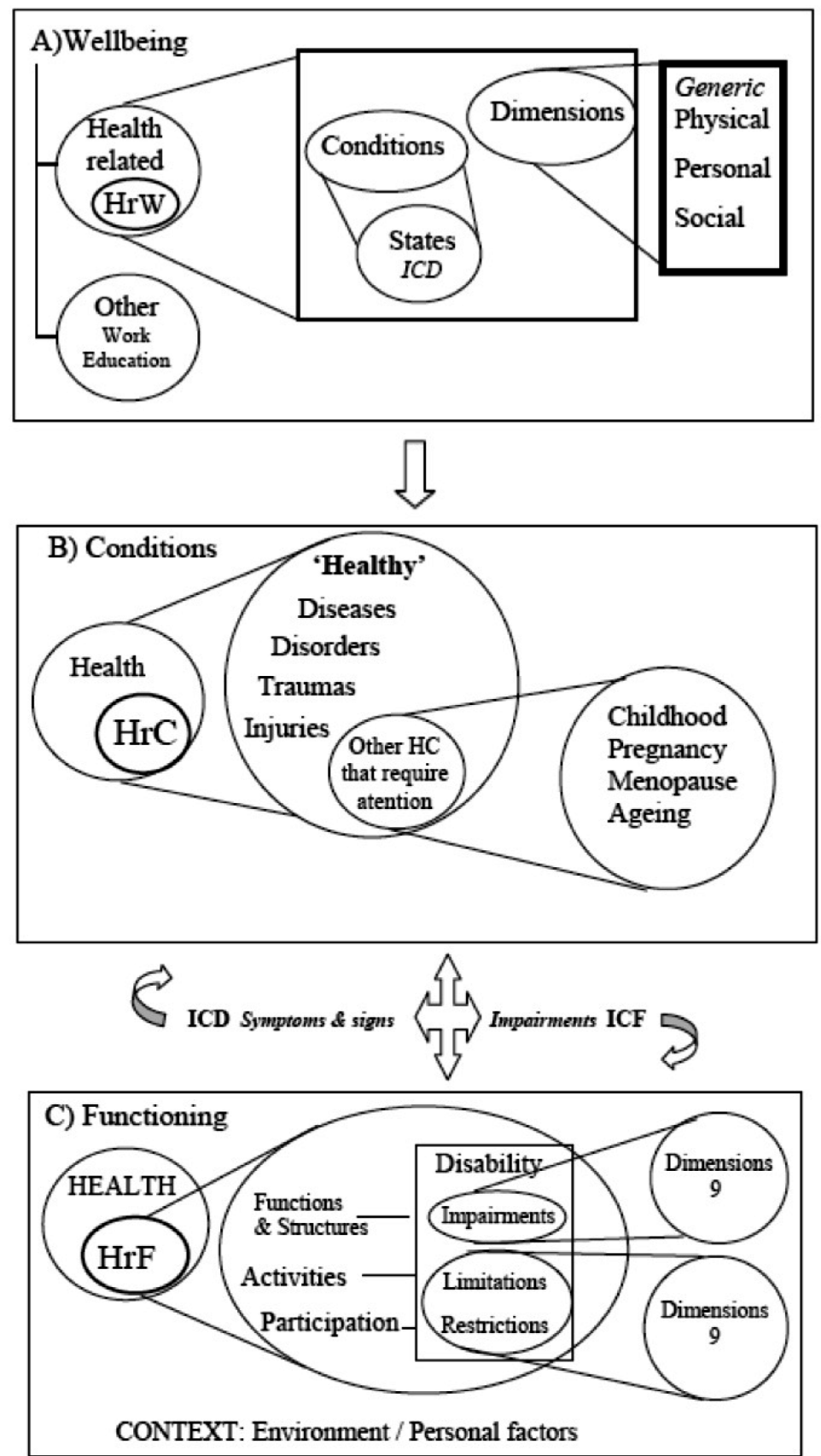

Figure 1. A preliminary conceptual map of A) Health related Wellbeing (HrW), B) Health related Conditions ( $\mathrm{HrC})$ and C) Health-related Functioning $(\mathrm{HrF})$ and disability including several perspectives from WHO: Health promotion, Health-related Quality of Life, and Health-related functioning based on the International Classification of Functioning, Disability and Health (ICF). 
wellbeing while HRQoL comprised all subjective aspects of $\mathrm{HrWB}$, including subjective functioning and disability.

However the construct (environmental) functioning-disability has moved from being a mere component of QoL to be considered a full domain of HrWB, together with its subjective aspects. Therefore, self-reported scales of functioning (e.g. WHODAS-II) are clearly part of the HrF construct and not part of the HRQoL one. On the other hand the "personal context" comprises traditional domains of QoL apart from other aspects. In the ICF model individual preferences and selfperceived status can be adequately differentiated while current QoL tends to confound these two personal factors. Therefore HRQoL has moved from Box "A" (all subjective wellbeing) to Box "C" (specific personal factors related to subjective experiences of wellbeing). On the other hand and due to the lack of development of the "personal context" domain at the 2001 version of ICF, it is now possible to develop this domain by incorporating the non-functional non-environmental aspects of HRQoL in the conceptual map of personal factors of ICF. In the future the "personal context" should be developed as an independent classification system that may encompass sociodemographics, personality, health related habits, health experiences, personal health determinants, life events and other personal factors that now are just mentioned at the ICF and it should be based -at least in part- on the existing evidence on QoL.

Within this future classification "health experiences" may play a central role. The boundaries and overlaps of the ICF-HrF model of health experience and the WHOQOL have been analysed by Cieza \& Stucki ${ }^{77}$. Their content analysis indicates that the ICF approach is broader, more comprehensive and embeds the QoL construct. In any case consensus within WHO is urgently needed re- garding the relation between HrWB and HRQoL. By now differences seem to broaden instead of being reduced. For instance, the development process of the WHOQOL disability module $\mathrm{e}^{42}$ has not been carried out through cooperation between these WHO groups and related networks (e.g. FDRG, Technical Advisory Group, WHOQOL-DIS Group $)^{78}$.

\section{Perspectives on Wellbeing from international projects}

The European MHADIE project (Measuring Health and Disability in Europe: supporting policy development) was very relevant in order to understand the role of $\mathrm{HrF}$ and QoL as components of HrWB in disabilities. HrF has been explored using a series of instruments closely related to ICF. The group defined "disability" as "a difficulty in functioning at the body, person or societal level, in one or more domains, as experienced by an individual with a health condition in interaction with contextual factors"79. The analysis of patients in specific groups indicates that the ICF-HrF model provides not only a better description of the disability associated to every health condition, but also a more comprehensive analysis of the individual wellbeing. The relationship between F\&D and QoL has been analysed in migraine patients $^{80}$, stroke survivors ${ }^{81}$ or in patients with multiple sclerosis ${ }^{82}$. Inconsistencies in the definition of disability were identified in previous studies comparing QoL and $\mathrm{HrF}$ in the latter group ${ }^{82}$.

Other relevant European projects related to wellbeing are the Collaborative Research on Ageing in Europe (COURAGE in Europe) and A Roadmap for Mental Health Research in Europe (ROAMER) projects. COURAGE in Europe project aimed to answer to the pressing need of the European Commission to have valid and reliable measures to de- 
scribe population ageing, which was identified among the most pressing policy issues in the $21^{\text {st }}$ century. To understand the effect of health on wellbeing and the impact of wellbeing on current and future health, it is essential that the notion of wellbeing is conceptualized and measured differently from the notion of health. The study incorporated an assessment of experiential and evaluative components of wellbeing. Experienced wellbeing was assessed with an abbreviated version of the Day Reconstruction Method ${ }^{83,84}$. The study also includes questions on evaluative wellbeing in order to examine which of these measures is more closely related to health and health related outcomes associated with ageing in different populations.

ROAMER project aims to develop an effective and widely accepted roadmap on the promotion and integration of mental health and wellbeing research in Europe ${ }^{85}$. ROAMER reflects a multi-disciplinary perspective, based on a consensus between the key stakeholders, using a methodologically sound, pragmatic, and comprehensive approach. One of the main objectives of ROAMER is to develop an accurate picture of the state-of-art in research on mental health and wellbeing in Europe. It also aims at establishing research priorities in wellbeing research, for the short, middle and long-term and applicable across the life span. The investigators of the project feel that the notion of wellbeing needs to be carefully conceptually defined because it is used in a perplexingly variety of inconsistent ways.

\section{The person centered approach to the "Health Experience"}

Person-centered medicine (PCM) places the person as a whole at the center of health care. The Person-centered Integrative Diagnosis (PID) is a PCM model aimed at facilitating person-centered care. According to this model, diagnosis is both a process and a formulation, and it should be oriented by the articulation of science and humanism, the use of a bio-psycho-social-cultural-spiritual framework. It should ensure engagement, empathy and partnership in the clinical care process, and sustain the patients' autonomy, responsibility and dignity while advancing the recovery and promotion of wellbeing. The key structural features of PID include the coverage of both positive and ill aspects of health, the person's experience and values, and the determinants of health. PID uses descriptive categories, dimensions, and narratives, to cultivate patient-family-clinician partnerships for achieving shared diagnostic understanding and shared commitment to care ${ }^{86}$.

The mental capital model has significant implications for the development of personcentered care strategies ${ }^{87}$. Mental capital "encompasses a person's cognitive and emotional resources. It includes their cognitive ability, how flexible and efficient they are at learning, and their "emotional intelligence", such as their social skills and resilience in the face of stress. It therefore conditions how well an individual is able to contribute effectively to society, and also to experience a high personal quality of life" 88 . The Foresight Project on Mental Capital and Wellbeing promotes mental capital initiatives in the population and in the healthcare system. The project tracks the implications of future challenges to our mental development along the life span and is intended to inform policy-makers both in the United Kingdom and around the world ${ }^{89}$.

\section{Conclusion}

During the last 30 years QoL has been a booming area in medicine and played a central role in understanding the contextual fac- 
tors of disease and illness. Paradoxically, new perspectives on QoL have led to a shift of interest from the 1990s simple indexes of QoL and from the generic and disease-specific QoL instruments, to an integrated perspective of individual wellbeing and its different components, including happiness, and functioning and disability. To some extent, the broader interest on health as a complex phenomenon has challenged the classical concept of HRQoL and facilitated the development of the broader field of HrWB, where different groups of models coexist and overlap (clinical, public health, economical, psychosocial and cultural, biopsychological, integrative). From a health terminology point of view it would be better to name this construct "Health related Being", but the term wellbeing is extended and it will be difficult to change.

From a dimensional perspective, health related wellbeing is an overarching construct characterised by asymmetrical polarity, where wellbeing embeds the concept of "ill-being" as health incorporates de concept of disease. The mental health group at International College for Person-centered Medicine has developed a bipolar dimensional model of the domains of health within the framework of person centered medicine ranging from ill health / illbeing to good health / wellbeing ${ }^{86}$ that incorporates all major aspects of wellbeing in its preliminary conceptual map: positive and negative polarity, condition status and functioning, experiences of health and contributors to ill and to good health.

A comprehensive conceptual map of a series of health related "meta-categories" (wellbeing, health condition, human functioning, disease, disability, etc.) is needed. Ideally, this mapping should include operational consensus-base definitions, the conceptual hierarchy of these entities and the granularity of their different domains, dimensions and facets. Although health ontology may shortly allow convergence of diverse approaches in this area, different meanings for the same concepts, and different names for related concepts will inevitably persist. Under these circumstances it becomes essential to understand the origin, evolution and current use of the different terms by different disciplines and professionals, and to build a "synset" (formal grouping of synonyms and semantically similar terms) that may facilitate both transfer across different sectors and semantic interoperability for care management and planning. Recently a similar "polynomicpolysemic" approach has been suggested for the use of the terms 'intellectual disability' and 'intellectual developmental disorders' in the health and social sectors ${ }^{90}$.

\section{Acknowledgements}

The research leading to these results has received funding from the European Community's Seventh Framework Programme under grant agreement numbers 223071 (COURAGE in Europe) and 282586 (ROAMER), from the Instituto de Salud Carlos III-FIS research grant number PS09/00295, and from the Spanish Ministry of Science and Innovation ACI-Promociona (ACI2009-1010 and ACI2011-1080). The study was supported by the Centro de Investigación Biomédica en Red de Salud Mental (CIBERSAM), Instituto de Salud Carlos III.

\section{References}

1. Colquhoun $\mathrm{D}$. The $\mathrm{A}$ to $\mathrm{Z}$ of the wellbeing industry: from Angelic Reiki to patient centred care. Wellbeing is big business, but how much of it works? BMJ 2011; 342: d2711. 
2. Angner E. The evolution of eupathics: the historical roots of subjective measures of wellbeing. International Journal of Wellbeing 2011; 1(1): 4-41.

3. Hart H. Chart for happiness. New York: Macmillan; 1940.

4. Diener E, Suh EM, Lucas RE, Smith HL. Subjective Well-Being: Three Decades of Progress. Psychol Bull 1999; 125(2): 276-302.

5. Kuznets S. National income and capital formation, 1919-1935. New York: National bureau of economic research; 1937.

6. Ayuso-Mateos JL, Salvador-Carulla L, Chisholm D. Use of quality of life measures in mental health economics and care planning. Actas Esp Psiquiatr 2006; 34(1): 1-6.

7. Tversky A, Kahneman D. The framing of decisions and the psychology of choice. Science 1981; 211(4481): 453-458.

8. Kahneman D. Objective happiness. In: Kahneman D, Diener E, Schwarz N, editors. Well-being: the foundations of hedonic psychology. New York: Russell Sage Foundation; 1999. p. 3-25.

9. Anielski M. The economics of happiness: building genuine wealth. Gabriola Island, BC: New Society Publishers; 2007.

10. Commission of the European Communities. GDP and beyond. Measuring progress in a changing world. 2009.

11. Stiglitz JE, Sen A, Fitoussi JP. Report by the Commission on the Measurement of Economic Performance and Social Progress. 2009.

12. Self A, Thomas J, Randall C. Measuring National Well-being: Life in the UK, 2012. Office for National Statistics, 2012.

13. Tobgay T, Dophu U, Torres CE, Na-Bangchang K. Health and Gross National Happiness: review of current status in Bhutan. J Multidiscip Healthc 2011; 4: 293-298.

14. Tobgay T, Dorji T, Pelzom D, Gibbons RV. Progress and delivery of health care in Bhutan, the Land of the Thunder Dragon and Gross National Happiness. Trop Med Int Health 2011; 16(6): 731-736.

15. Pennock M, Ura K. Gross national happiness as a framework for health impact assessment. Environ Impact Assess Rev 2011; 31(1): 61-65.

16. Scorsolini-Comin F, dos Santos MA. The scientific study of happiness and health promotion: an integrative literature review. Rev Lat Am Enfermagem 2010; 18(3): 472-479.

17. Gallup. Gallup-Healthways Well-being Index: Methodology Report for Indexes. Gallup Inc., 2009.

18. Political and Economic Planning. Report on the British health services: a survey of the existing health ser- vices in Great Britain, with proposals for future development. London: Political and Economic Planning; 1937.

19. Horder T. Health with happiness - Lord Horder's speech at Leeds. BMJ 1937; 2: 858-859.

20. Tejada de Rivero DA. Alma Ata revisited. Perspect Health 2003; 8 (2): 3.

21. Mattei C. [IX. International Hospital Congress; mental wellbeing of the patient]. Tech Hosp Med Soc Sanit. 1955; 10(118): 47-49.

22. Linder FE. The health of the American people. Sci Am 1966; 214(6): 21-29.

23. Bradburn NM. The structure of psychological wellbeing. Chicago: Aldine; 1969.

24. Prutkin JM, Feinstein AR. Quality-of-life measurements: origin and pathogenesis. Yale J Biol Med 2002; 75(2): 79-93.

25. Gershuny J. Time-use surveys and the measurement of national well-being. Centre for time-use research. Department of sociology. University of Oxford, 2011.

26. Karnofsky DA, Burchenal JH. The clinical evaluation of chemotherapeutic agents in cancer. In: Macleod CM, editor. Evaluation of Chemotherapeutic Agents. New York: Columbia University Press; 1948.

27. Katz S, Ford AB, Moskowitz RW, Jackson BA, Jaffe MW. Studies of illness in the aged. The index of ADL: A standardized measure of biological and psychosocial function. JAMA 1963; 185: 914-919.

28. Campbell A, Converse PE. The human meaning of social change. New York: Rusell Sage Foundation; 1972.

29. Andrews FM, Withey SB. Social Indicators of WellBeing. London: Plenum Publishers; 1976.

30. Carlens E, Dahlstrom G, Nou E. Comparative measurements of quality of survival of lung cancer patients after diagnosis. Scand J Respir Dis 1970; 51(4): 268-275.

31. Feinstein AR, Pritchett JA, Schimpff CR. The epidemiology of cancer therapy. II. The clinical course: data, decisions, and temporal demarcations. Arch Intern Med 1969; 123(3): 323-344.

32. Priestman TJ, Baum M. Evaluation of quality of life in patients receiving treatment for advanced breast cancer. Lancet 1976; 1(7965): 899-900.

33. Aaronson NK, Acquadro C, Alonso J, Apolone G, Bucquet D, Bullinger M, et al. International Quality of Life Assessment (IQOLA) Project. Qual Life Res 1992; 1(5): 349-351.

34. The WHOQOL group. Development of the WHOQOL: rationale and current status. Int J Ment Health 1994; 23: $24-56$. 
35. WHOQOL group. Study protocol for the World Health Organization project to develop a Quality of Life assessment instrument (WHOQOL). Qual Life Res 1993; 2(2): 153-159.

36. Kuyken W, Orley J. Development of the WHOQOL - Rationale and Current Status. Int J Ment Health 1994; 23(3): 24-56.

37. Kuyken W, Orley J, Power M, Herrman H, Schofield $\mathrm{H}$, Murphy B, et al. The World-Health-Organization Quality-Of-Life Assessment (WHOQOL) - Position Paper from the World-Health-Organization. Soc Sci Med 1995; 41(10): 1403-1409.

38. Power M, Harper A, Bullinger M. The World Health Organization WHOQOL-100: tests of the universality of Quality of Life in 15 different cultural groups worldwide. Health Psychol 1999; 18(5): 495-505.

39. Power M, Kuyken W, Orley J, Herrman H, Schofield H, Murphy B, et al. The World Health Organization Quality of Life assessment (WHOQOL): Development and general psychometric properties. S Soc Sci Med 1998; 46(12): 1569-1585.

40. WHOQOL SRPB Group. A cross-cultural study of spirituality, religion, and personal beliefs as components of quality of life. Soc Sci Med 2006; 62(6): 1486-1497.

41. Power M, Quinn K, Schmidt S, WHOQOL-OLD Group. Development of the WHOQOL-old module. Qual Life Res 2005; 14(10): 2197-2214.

42. Power M, Green AM, WHOQOL-Dis Group. Development of the WHOQOL disabilities module. Qual Life Res 2010; 19(4): 571-584.

43. Aaronson NK. Methodologic issues in assessing the quality of life of cancer patients. Cancer 1991; 67(3 Suppl): 844-850.

44. Chisholm D, Salvador-Carulla L, Ayuso-Mateos JL. Quality of life measurement in the economic analysis of mental health care. In: Katschnig H, Freeman H, Sartorius N, editors. Quality of life in mental disorders, 2nd ed. New York: John Wiley and Sons Inc; 2006. p. 299-308.

45. Weinstein MC, Stason WB. Foundations of cost-effectiveness analysis for health and medical practices. N Engl J Med 1977; 296(13): 716-721.

46. Donaldson C, Baker R, Mason H, Jones-Lee M, Lancsar E, Wildman J, et al. The social value of a QALY: raising the bar or barring the raise? BMC Health Serv Res 2011; $11: 8$.

47. Gillham JE, Seligman ME. Footsteps on the road to a positive psychology. Behav Res Ther 1999; 37 Suppl 1: S163-S173.
48. Ryff CD. Happiness Is Everything, Or Is It - Explorations on the Meaning of Psychological Well-Being. J Pers Soc Psychol 1989; 57(6): 1069-1081.

49. Ryff CD, Singer B. Psychological well-being: meaning, measurement, and implications for psychotherapy research. Psychother Psychosom 1996; 65(1): 14-23.

50. Ryff CD, Magee WJ, Kling KC, Wing EH. Forging macro-micro linkages in the study of psychological wellbeing. In: Ryff CD, Marshall VW, editors. The self and society in aging process. New York: Springer; 1999. p. 247-278.

51. Ryan RM, Deci EL. On happiness and human potentials: a review of research on hedonic and eudaimonic well-being. Annu Rev Psychol 2001; 52: 141-166.

52. Seligman ME, Csikszentmihalyi M. Positive psychology. An introduction. Am Psychol 2000; 55(1): 5-14.

53. Singer B, Friedman E, Seeman T, Fava GA, Ryff CD. Protective environments and health status: cross-talk between human and animal studies. Neurobiol Aging 2005; 26 Suppl 1: 113-118.

54. Schalock RL, Keith KD, Hoffman K, Karan OC. Quality of life: its measurement and use. Ment Retard 1989; 27(1): 25-31.

55. Wang M, Schalock RL, Verdugo MA, Jenaro C. Examining the factor structure and hierarchical nature of the quality of life construct. Am J Intellect Dev Disabil 2010; 115(3): 218-233.

56. Frank AW. What is dialogical research, and why should we do it? Qual Health Res 2005; 15(7): 964-974.

57. Morrow MR. Feeling unsure: a universal lived experience. Nurs Sci Q 2010; 23(4): 315-325.

58. Jonas-Simpson CM. Feeling understood: a melody of human becoming. Nurs Sci Q 2001; 14(3): 222-230.

59. Pilkington FB, Kilpatrick D. The lived experience of suffering: a parse research method study. Nurs Sci Q 2008; 21(3): 228-237.

60. Gray JA. The psychophysiological basis of introversion-extraversion. Behav Res Ther 1970; 8(3): 249-266.

61. Cloninger CR, Svrakic DM, Przybeck TR. A psychobiological model of temperament and character. Arch Gen Psychiatry 1993; 50(12): 975-990.

62. Cloninger CR. Feeling good: the science of wellbeing. Oxford: Oxford University Press; 2004.

63. Cloninger CR. The science of well-being: an integrated approach to mental health and its disorders. World Psychiatry 2006; 5(2): 71-76.

64. Josefsson K, Cloninger CR, Hintsanen M, Jokela M, Pulkki-Raback L, Keltikangas-Jarvinen L. Associations of personality profiles with various aspects of well-being: a population-based study. J Affect Disord 2011; 133(1-2): 265-273. 
65. Salloum IM, Mezzich JE. Outlining the bases of person-centred integrative diagnosis. J Eval Clin Pract 2011; 17(2): 354-356.

66. Herrman H, Saxena S, Moodie R, editors. Promoting mental health: concepts, emerging evidence, practice. Geneva: World Health Organization; 2005.

67. Hosman C, Jane-Llopis E, Saxena S, editors. Prevention of mental disorders: effective interventions and policy options. Oxford: Oxford University Press; 2005.

68. World Health Organization. Health Promotion Glossary. Geneva: World Health Organization; 1998.

69. World Health Organization. Ageing. Exploding the myths. World Health Organization; 1999.

70. World Health Organization. Ottawa charter for health promotion. World Health Organization; 2013.

71. World Health Organization. International Classification of Functioning, Disability and Health: ICF. Geneva: World Health Organization; 2001.

72. World Health Organization. International Classification of Health Interventions (ICHI). Geneva: World Health Organization; 2010.

73. OECD, Eurostat, WHO. A system of health accounts: OECD Publishing; 2011.

74. Salvador-Carulla L, Garcia-Gutierrez C. The WHO construct of health-related functioning $(\mathrm{HrF})$ and its implications for health policy. BMC Public Health 2011; 11 Suppl 4: S9.

75. Kumar A, Smith B, editors. The ontology of processes and funtions: a study of the international classification of functioning, dissability and health. AIME 2005 Workshop on Biomedical Ontology Engineering; 2005.

76. Della Mea V, Simoncello A, editors. Onto ICF: a first report on ICF and SUMO. Meeting of the WHO Collaborating Centres For The Family of International Classifications; 2009.

77. Cieza A, Stucki G. The International Classification of Functioning Disability and Health: its development process and content validity. Eur J Phys Rehabil Med 2008; 44(3): 303-313.

78. Salvador-Carulla L, Gasca VI. Defining disability, functioning, autonomy and dependency in person-centered medicine and integrated care. Int J Integr Care 2010; 10 Suppl: e025.

79. Raggi A, Leonardi M, Cabello M, Bickenbach JE. Application of ICF in clinical settings across Europe. Disabil Rehabil 2010; 32 Suppl 1: S17-S22.

80. Leonardi M, Raggi A, Bussone G, D'Amico D. Health-related quality of life, disability and severity of disease in patients with migraine attending to a specialty headache center. Headache 2010; 50(10): 1576-1586.
81. Geyh S, Cieza A, Kollerits B, Grimby G, Stucki G. Content comparison of health-related quality of life measures used in stroke based on the international classification of functioning, disability and health (ICF): a systematic review. Qual Life Res 2007; 16(5): 833-851.

82. Svestkova O, Angerova Y, Sladkova P, Keclikova B, Bickenbach JE, Raggi A. Functioning and disability in multiple sclerosis. Disabil Rehabil 2010; 32 Suppl 1: S59-S67.

83. Ayuso-Mateos JL, Miret M, Caballero FF, Olaya B, Haro JM, Kowal P, et al. Multi-country evaluation of affective experience: validation of an abbreviated version of the day reconstruction method in seven countries. PloS One 2013; 8(4): e61534.

84. Miret M, Caballero FF, Mathur A, Naidoo N, Kowal $\mathrm{P}$, Ayuso-Mateos JL, et al. Validation of a measure of subjective well-being: an abbreviated version of the day reconstruction method. PLoS One 2012; 7(8): e43887.

85. Haro JM, Ayuso-Mateos JL, Bitter I, DemotesMainard J, Leboyer M, Lewis SW, et al. ROAMER: roadmap for mental health research in Europe. Int J Methods Psychiatr Res 2014 Jan; 23 Suppl 1: 1-14.

86. Mezzich JE, Snaedal J, van Weel C, Heath I. Introduction to conceptual explorations on person-centered medicine. Int J Integr Care 2010; 10: e002.

87. Salvador-Carulla L, Ruiz M, Saavedra JE. Promoting wellbeing in persons with disabilities. The international journal of person centered medicine 2012; 2(1).

88. Foresight Mental Capital Wellbeing Project. Final project report - executive summary. London: The Government Office for Science; 2008.

89. Beddington J, Cooper CL, Field J, Goswami U, Huppert FA, Jenkins R, et al. The mental wealth of nations. Nature 2008; 455(7216): 1057-1060.

90. Salvador-Carulla L, Reed GM, Vaez-Azizi LM, Cooper SA, Martinez-Leal R, Bertelli M, et al. Intellectual developmental disorders: towards a new name, definition and framework for "mental retardation/intellectual disability” in ICD-11. World Psychiatry 2011; 10(3): 175-180.

\author{
Corresponding author: \\ Luis Salvador-Carulla \\ Centre for Disability Research and Policy \\ Faculty of Health Sciences \\ T315a Cumberland Campus \\ The University of Sydney \\ 75 East Street I Lidcombe I NSW I 2141, Australia \\ Tel: +61-293 519231 \\ Fax: +61-293 519566 \\ E-mail: luis.salvador-carulla@sydney.edu.au
}

